\section{¿Cosmopolitismo o} subordinación? La participación de científicos latinoamericanos en programas europeos: motivaciones y dinámicas analizadas desde el punto de vista de los líderes europeos

\section{Cosmopolitanism or}

subordination? Latin American scientists' participation in European programs: motivations and dynamics analyzed from the European scientific leaders' point of view

\section{Adriana Feldi}

' Investigadora, Consejo Nacional de Investigaciones Científicas y Técnicas. Buenos Aires - Argentina

feldri75@yahoo.com.mx

orcid.org/0000-0002-9857-746X

\section{Pablo Kreimeri}

ii Investigador, Consejo Nacional de Investigaciones Científicas y Técnicas; director, Centro de Ciencia, Tecnología y Sociedad/Universidad Maimónides. Buenos Aires - Argentina

pkreimer@yahoo.com

orcid.org/0000-0002-6737-8556

Recebido em 6 dez. 2017.

Aprovado em 15 mar. 2018.
FELD, Adriana; KREIMER, Pablo. ¿Cosmopolitismo o subordinación? La participación de científicos latinoamericanos en programas europeos: motivaciones y dinámicas analizadas desde el punto de vista de los líderes europeos. História, Ciências, Saúde - Manguinhos, Rio de Janeiro, v.26, n.3, jul.-set. 2019, p.779-799.

Resumen

Partiendo de un enfoque cualitativo, pretendemos dar cuenta de las motivaciones de grupos científicos y sus dinámicas de cooperación internacional. Consideramos la participación de científicos latinoamericanos en consorcios europeos y analizamos el punto de vista de los líderes europeos. Dentro de la cooperación científica "Norte-Sur", planteamos tres niveles de análisis: a nivel institucional, comprender el interés de Europa en cooperar con Latinoamérica; en relación con las estrategias cognitivas, averiguar las motivaciones de líderes europeos para enrolar latinoamericanos en sus investigaciones; a nivel de las prácticas, conocer la organización del trabajo en el interior de esos consorcios. Hacia el final, proponemos una tipología de algunas configuraciones de cooperación científica de grupos latinoamericanos.

Palabras clave: cooperación científica internacional; centros y periferias; Europa y América Latina.

\section{Abstract}

Using a qualitative approach, we explore the motivations of scientific groups and their dynamics in international cooperation. We consider the participation of Latin American scientists in European consortia and analyze the European hosts' point of view. Within the framework of "NorthSouth" scientific cooperation, we propose three levels of analysis: on the institutional level, understanding Europe's interest in cooperating with Latin America; in terms of cognitive strategies, tracing what motivates European group leaders to include Latin Americans in their research projects; and in terms of practices, figuring out how work within these consortia is allotted. Towards the end, we propose a typology of some of the modalities of scientific cooperation by Latin American groups.

Keywords: international scientific cooperation; centers and peripheries; Europe and Latin America. 
$\mathrm{L}$ a cooperación entre científicos de diversos países no ha cesado de aumentar, en particular en las últimas 3 décadas, lo que se ha constatado a través de las publicaciones (Adams, 2012, 2013). Algunos autores, como Leydesdorf y Wagner (2009) señalan que "medidos por las coautorías, hay un crecimiento lineal entre 1990 y 2005 en términos del número de papers", pero creció en forma "exponencial en términos del número de domicilios internacionales", lo que confirmaría la hipótesis de una "inflación" de las colaboraciones internacionales (Persson, Glänzel, Danell, 2004). En efecto, la proporción de artículos escritos en coautoría internacional versus los artículos "domésticos" es cada vez mayor en un conjunto importante de países (en el Reino Unido o en Suiza, es más de la mitad; ver Adams, 2013). Así en 1988, poco más de un 10\% de los papers estaban firmados por investigadores de más de un país, pero veinte años más tarde ese porcentaje había ascendido al 30\% (Boekholt et al., 2009; Gaillard, Gaillard, Arvanitis, 2010). En ese contexto, no sorprende constatar que la participación de científicos latinoamericanos en proyectos desarrollados con grupos y colegas de países más desarrollados ha aumentado en forma muy significativa durante ese período (Gaillard, Arvanitis, 2013; Kreimer, Levin, 2014). Por ello, vale la pena preguntarnos acerca de las formas que adquiere dicha colaboración, particularmente en términos cualitativos, considerando las percepciones de los líderes europeos.

Haremos, en este artículo, cuatro recortes significativos dentro del tópico general de la cooperación internacional. En primer lugar, nos interesa analizar la cooperación que generalmente se ha denominado "Norte-Sur", o entre países centrales y contextos semiperiféricos o "no hegemónicos" (Losego, Arvanitis, 2008; Kreimer, 2006). ${ }^{1}$ Segundo, nos interesa observar, dentro de este tipo de relaciones, a la cooperación científica entre América Latina y Europa, por razones que explicaremos enseguida. Tercero, si bien somos conscientes de que no todas las relaciones de cooperación internacional se dan bajo un paraguas de programas de cooperación formal, nos concentraremos aquí en los proyectos institucionalizados cuyo financiamiento lo provee la Unión Europea y en donde participan grupos latinoamericanos. Cuarto, a diferencia de la mayor parte de los estudios sobre cooperación internacional, no trabajamos sobre publicaciones (coautorías $\mathrm{u}$ otros indicadores) sino que proponemos un estudio cualitativo sobre las percepciones y motivaciones de los actores involucrados.

En un artículo reciente hemos investigado el punto de vista de los líderes latinoamericanos, indagando las percepciones y actitudes de los investigadores de la región que han participado en proyectos europeos (Kreimer, Levín, 2014). ${ }^{2}$ Complementariamente, en el presente texto mostraremos "la otra cara de la moneda", concentrándonos exclusivamente en las perspectivas de los líderes europeos (aspecto que aparece con mucha menos frecuencia en la literatura), con la expectativa de combinar ambas miradas en un texto futuro. Para eso, seleccionamos un conjunto de proyectos del séptimo Programa Marco, en los que participaron grupos de investigación de los cinco países más dinámicos de América Latina (Argentina, Brasil, México, Chile y Colombia). La elección de estos cinco países se debe a que, en su conjunto, representan más del 80\% de la participación latinoamericana en consorcios europeos. Como veremos, aunque América Latina no representa un objetivo específico dentro de la política europea de cooperación con terceros países, el incremento de la participación latinoamericana en 
los Programas Marco (por motivos que expondremos más adelante) explica la relevancia de estudiar este tipo de cooperación birregional.

La pregunta general que guía este artículo se orienta a comprender por qué los consorcios europeos incorporan grupos de investigación de América Latina. Ello se declina, a su vez, en tres preguntas: a) en el plano político-institucional ¿cuál es el interés de la Unión Europea en fomentar la cooperación con América Latina? b) en el plano de las estrategias cognitivas ¿cuáles son las motivaciones de los líderes de proyectos europeos para incluir grupos de América Latina en la conformación de los consorcios de investigación? y c) en el plano de las prácticas ¿cómo describen los líderes europeos el papel de los grupos latinoamericanos en dichos consorcios?

Para responder a estos interrogantes, en la sección 1 discutimos los modos de abordaje y proporcionamos un conjunto de precisiones sobre el objeto de estudio, tomando en consideración dos elementos que nos permiten dar cuenta del primer interrogante: a) aspectos organizacionales y geopolíticos que nos permiten conceptualizar los tipos de cooperación; b) los sucesivos paradigmas de cooperación científica Norte-Sur que han guiado las políticas de los países desarrollados. En la sección 2, describimos la metodología utilizada. Por último, en la sección 3, presentamos los resultados y el análisis de la información obtenida, organizada en torno a dos variables: las motivaciones de los líderes europeos para enrolar a grupos latinoamericanos; y las actividades desarrolladas por estos últimos, dentro de la división interna del trabajo, así como los beneficios obtenidos. Allí también ofrecemos una tipología preliminar en función de las variables propuestas, para finalizar con algunas conclusiones.

\section{Abordaje de la cooperación científica internacional: algunas precisiones sobre nuestro objeto de estudio}

\section{Consideraciones generales sobre la cooperación científica internacional y sobre la cooperación Norte-Sur}

Las definiciones, alcance, actores, instituciones, regulaciones y prácticas específicas de la cooperación científica internacional han sido objeto de múltiples ambigüedades y debates. Por lo tanto, antes de adentrarnos en el análisis específico de nuestro objeto, es necesario establecer, por lo menos, dos precisiones referidas a la cooperación Norte-Sur y una vinculada con la cooperación científica internacional en términos generales, que nos permitirán delimitar los perfiles de nuestro objeto.

La cooperación Norte-Sur presenta problemas y tensiones interpretativas específicas. En primer lugar, por la definición misma de los actores en juego: algunos trabajos sobre cooperación Norte-Sur se refieren a los "países en desarrollo" como a un conjunto relativamente homogéneo de países. Así, por ejemplo, Wagner señala que con la emergencia de las nuevas redes como modo de organizar la producción del conocimiento en el siglo XXI, existen varias operaciones en los usos del conocimiento que permanecen en la esfera local, por lo que los países en desarrollo necesitan establecer y desarrollar una serie de arreglos institucionales si quieren sacar ventaja de estas posibilidades. Según Wagner (2008, p.115), 
"como esos países no tienen burocracias propias del siglo XX ni instituciones que fueron la marca de la era del nacionalismo científico, tienen gran flexibilidad para emprender nuevos desarrollos en la ciencia. La ausencia de restricciones basadas en lo nacional, junto con grandes inversiones, puede realmente ser una ventaja que los países en desarrollo pueden explotar, estableciendo un sistema de red más ágil".

El problema de esta perspectiva es que bajo la etiqueta de "países en desarrollo" se incluyen solo aquellos con escasas tradiciones científicas o con sistemas nacionales de ciencia débiles. Sin embargo, en términos de producción de conocimientos, países como Argentina, Chile, Brasil, México, Egipto, Sudáfrica, entre otros que pertenecen al amplio "mundo en desarrollo", son altamente dinámicos y disponen de sistemas científicos hace más de un siglo, como mínimo. En ese sentido, Gaillard, Gaillard y Arvanitis (2010) mostraron que en América Latina la performance de la cooperación internacional es muy diferente según la fortaleza científica de cada país. Por lo tanto, para este trabajo hemos escogido países latinoamericanos con niveles de desarrollo (socio-económico y científico) relativamente similares que nos permite hacer inferencias sobre bases más sólidas.

En segundo lugar, la cooperación científica "Norte-Sur" suele ser presentada por los tomadores de decisiones, tanto europeos como latinoamericanos, como algo "bueno en sí mismo", con un discurso dual: por el lado de los latinoamericanos, ello está impregnado de cosmopolitismo, de las ventajas de interactuar con los líderes mundiales y de dar mayor visibilidad a la ciencia local. Por el lado de la Unión Europea, los discursos son más pragmáticos y se refieren a la cooperación como un recurso estratégico para fortalecer la ciencia y, sobre todo, la competitividad de Europa.

Sin embargo, la literatura sobre el tema pone de relieve ciertas tensiones interpretativas, en particular entre visiones "optimistas" versus visiones críticas. Algunos trabajos se han hecho eco de esa mirada optimista, celebrando la intensificación de la cooperación como un modo de acrecentar el cosmopolitismo de los investigadores del "Sur" e integrarlos en la "ciencia internacional" (Sebastian, 2007), o bien, subrayando el efecto democratizador de las nuevas formas de cooperación internacional y las "oportunidades" que se presentan para los países en desarrollo (Wagner, 2008; Anderson, 2011). Por otro lado, las perspectivas más críticas han puesto el énfasis en las asimetrías y las relaciones de subordinación o, incluso, de dependencia, que estructuran los modos de colaboración entre contextos de mayor y menor desarrollo relativo (Gaillard, 1994; Vessuri, 1996; Cetto, Vessuri, 2005; Velho, 2002; Kreimer, 1998; Kreimer, Meyer, 2008; Kreimer, Levin, 2014; Beigel, 2014). Lo que está en juego es si esa cooperación mejora las capacidades técnicas y cognitivas de los países en desarrollo, si se trata de un modo de inserción marginal y subordinada, si existen asimetrías en la definición de las agendas de investigación y si esas agendas se adecuan - o no - a las necesidades de los países en desarrollo. Este trabajo explora esta cuestión a través de una estrategia metodológica que enfoca las dinámicas de trabajo al interior de los consorcios de investigación, de modo de dar cuenta de la naturaleza compleja - y a menudo contradictoria - de las relaciones de cooperación internacional entre grupos de diferente desarrollo relativo.

En lo referente a la cooperación científica internacional en términos más amplios, Katz y Martin (1997) han señalado en su momento la ambigua definición de aquello que 
entendemos por cooperación. Por ejemplo, una abundante literatura toma como material para el estudio de las relaciones de cooperación internacional a las publicaciones científicas - perspectiva bibliométrica - y, en particular, las coautorías, pues se supone que representan una buena proxy de dichas prácticas (Gläser, Laudel, 2001; Newman, 2001; Leydesdorff, Wagner, 2009; Wagner, Kit Wong, 2012). Aunque esta metodología macro permite observar cierta dinámica en términos de grandes tendencias de cooperación en determinados países, en campos disciplinarios específicos e, incluso, en núcleos de concentración temática, solo muestra una parte del producto de las colaboraciones (los papers) y no nos dice nada sobre las modalidades de organización y las motivaciones para la cooperación, que son aspectos cruciales para comprender las relaciones científicas entre centros y periferias. De hecho, muchas actividades y prácticas sociales no se cristalizan necesariamente en artículos científicos, sino que se dan de muchas otras formas, como el intercambio de investigadores y becarios, el desarrollo de programas conjuntos de formación, el desarrollo de productos para la industria, la organización de seminarios etc. (Rodríguez Medina, 2014).

En términos generales, podemos decir que hay tres elementos contextuales que explican algunas de las transformaciones en la organización de la cooperación en las últimas décadas. El primero de ellos refiere a los cambios de escala que se fueron produciendo desde el final del siglo XX, cuando se fue pasando de la llamada big science (Price, 1973; Galison, Hevly, 1992), tal como se fue desplegando desde la posguerra y la guerra fría (Hallonsten, 2016), hacia una escala mucho mayor, una de cuyas expresiones - pero no la única - son diversos "mega emprendimientos" (Beaver, 2001; Wagner, 2008; Kreimer, 2012) como el secuenciamiento del genoma humano o el Large Hadron Collider, que movilizaron a miles de investigadores. A diferencia del modelo de big science tradicional, que desde los países en desarrollo ha estado asociado con el brain drain (Devan, Tewari, 2001; OECD, 2002), estos proyectos no implican necesariamente la radicación de muchos investigadores en un mismo lugar físico, sino que, gracias a la masificación de las TICs, los científicos se integran - bajo la forma de redes - desde diferentes localizaciones geográficas (Shrum, 2005; Adams, 2012).

La segunda tiene que ver con la reorganización de las disciplinas tradicionales y la emergencia de nuevos campos. En efecto, los espacios de investigación se tornaron más complejos y se generaron desplazamientos e hibridaciones entre disciplinas y también entre investigación académica e investigación industrial, en "comunidades de investigación tecnológica" (Joerges, Shinn, 2001), y nuevos regímenes de producción de conocimiento (Pestre, 2003, p.104).

La tercera se relaciona con las políticas de estímulo a la cooperación internacional, que han dado un impulso explícito a estos vínculos, tanto en los discursos como en la puesta en marcha de instrumentos específicos: la Unión Europea (European Commission, 2008) y los Estados Unidos (National Science Board, 2008) han dispuesto, desde hace varias décadas, instrumentos específicos para fomentar la cooperación internacional. Los países europeos lo promovieron tanto a nivel agregado como a nivel nacional, mientras que los Estados Unidos lo ha hecho de modo diversificado a través de numerosas instituciones y agencias (Whitley, 2010), tanto públicas como privadas.

Estos tres elementos son fundamentales para comprender la modalidad específica de cooperación a la que nos referimos en este trabajo. Se trata de una cooperación que resulta 
de políticas explícitas de la Unión Europea, orientada hacia objetivos preestablecidos en los programas de cada área temática y en las convocatorias. Eso implica la conformación de medianos o grandes consorcios internacionales, con una duración de 4 años aproximadamente, en los que cada grupo cumple un rol específico dentro del tópico o problemática en cuestión, trascendiendo las barreras disciplinarias. En ese sentido, es probable que muchas de las conclusiones que extraigamos en este estudio no sean generalizables a otras modalidades como la cooperación informal u ocasional, de menor escala o basada en proyectos específicos derivados de acuerdos binacionales.

Dado que las políticas de la Unión Europea tienen un rol fundamental en el tipo de cooperación que pretendemos analizar, es necesario distinguir, como ha hecho buena parte de la literatura, las motivaciones políticas de las motivaciones individuales para la cooperación y su articulación con otros factores que la estimulan o la dificultan (Beaver, 2001; Bozeman, Corley, 2004; Boekholt et al., 2009; Edler, Flanagan, 2011; Wagner, 2006, 2008; Gaillard, Arvanitis, 2013). Por lo tanto, en la sección siguiente analizamos algunas de las características generales de la política de cooperación extra-europea de los Programas Marco, que nos permitirá comprender mejor el rol de los investigadores latinoamericanos en los consorcios internacionales de investigación.

\section{La cooperación Norte-Sur y la política de cooperación internacional de la Unión Europea}

Tomando en consideración las políticas de cooperación científica de organismos internacionales y agencias de financiamiento localizadas en países desarrollados, Gaillard (1999) identifica tres fases sucesivas en la cooperación científica Norte-Sur: la primera, que se extiende desde el período colonial hasta las décadas de 1960-1970, se enfocó en encontrar soluciones rápidas al problema del desarrollo a través de la movilización de recursos (humanos y financieros) científicos de los países del Norte; la segunda, que transcurre entre la década de 1970 y las de 1980-1990, se centró en la construcción de capacidades endógenas en los países del Sur; la tercera, que es la más reciente, se orienta a generar estructuras de cooperación en las que el Leitmotiv es el beneficio mutuo.

Las políticas de cooperación científica de la Unión Europea con países en desarrollo han seguido criterios análogos a los descriptos por Gaillard. En efecto, dichas políticas se remontan a la década de 1980, cuando el Parlamento Europeo creó el Programa Ciencia y Tecnología para el Desarrollo (STD), que tuvo tres fases: STD I (1983-1987), STD II (19871990) y STD III (1991-1994). Estos programas tenían el propósito de reforzar las capacidades de investigación e incrementar el impacto de la investigación en los países en desarrollo, especialmente a través de campos como la agricultura tropical y subtropical, por un lado, y la medicina, la salud y la nutrición, por otro (Gaillard, 1994).

A partir del $4^{\text {o }}$ Programa Marco (1994-1998), la Unión Europea creó un sub-programa especial para gestionar la "Cooperación con Terceros Países y Organismos Internacionales" (INCO), que incluyó la cooperación con países en desarrollo (INCO-DC). El INCO-DC amplió el abanico de temáticas, incluyendo algunas no vinculadas estrictamente a las necesidades de los países en desarrollo: 1) gestión de recursos naturales renovables (florestas, océanos, agua, energía); 2) agricultura y agroindustria (mejoramiento de la producción, 
almacenamiento y comercialización); 3) salud (control de enfermedades, vacunas, sistemas de asistencia); 4) temas de interés mutuo establecidos de común acuerdo en sectores como las tecnologías de la información y la comunicación, nuevos materiales etc. (Gusmão, 2000).

A partir del sexto y, sobre todo, del séptimo Programa Marco, se produjo una novedad interesante: la cooperación con países en desarrollo no presenta diferencias significativas, en materia de instrumentos, respecto de la cooperación intra-europea o con países desarrollados (European Commission, 2005). Para que se entienda esta innovación, es necesario reseñar muy brevemente la estructura general del séptimo Programa Marco (muy similar al sexto), organizado en 4 subprogramas (European Commission, 2007):

a. Cooperación. Financia proyectos de consorcios internacionales, con participación europea y de terceros países en las siguientes áreas prioritarias: salud, agricultura pesca y ganadería, tecnologías de la información y la comunicación, nanociencias nanotecnologías y materiales, energía, medioambiente, transporte, ciencias socioeconómicas y humanas, espacio, seguridad.

b. Ideas. Financia investigación de excelencia de grupos individuales, que no necesariamente deben incorporar terceros países.

c. Personas. Financia proyectos de movilidad intra y extra-europea.

d. Capacidades. Contempla cuatro instrumentos de los que pueden participar terceros países: (a) infraestructura para investigación; (b) investigación para beneficio de pequeñas y medianas empresas; (c) regiones del conocimiento, potencial de investigación, la ciencia en la sociedad; e (d) actividades específicas de cooperación internacional (INCO).

Dentro de esta estructura, la cooperación con terceros países deja de canalizarse exclusivamente a través del subprograma INCO (que se transforma en una línea de financiamiento destinada a actividades de networking y construcción de agendas birregionales) y los terceros países también pueden participar en pie de igualdad con los estados miembros del subprograma "cooperación". Este último es el más relevante en términos presupuestarios, pues concentra un 69,5\% de los recursos del sexto Programa Marco y un 64,1\% del séptimo (ver <https://cordis.europa.eu/fp6/budget.htm>; European Commission, 2007).

Detrás de este cambio organizacional hay una enérgica política de fortalecimiento del "espacio europeo de investigación" (ERA), para el que se considera necesario estrechar las relaciones internacionales intra y extra-europeas. Esta política se expresó en un aumento del 40\% en el presupuesto anual del séptimo Programa Marco, respecto del sexto; una tendencia que continúa ascendente en el reciente Horizonte 2020 (Muldur et al., 2006). Esto explica, en buena medida, el incremento de la participación latinoamericana: en el sexto Programa marco (2002-2006), diversos grupos latinoamericanos participaron de 204 proyectos, mientras que en el séptimo (2007-2013) colaboraron con 308 proyectos (Eularinet, 2009; Cordis, 2009). ${ }^{3}$

Vale la pena preguntarse entonces qué motivaciones subyacen a esta política. En un esfuerzo de síntesis y clasificación de las motivaciones para las políticas de cooperación internacional, Boekholt et al. (2009) diferencian el "paradigma limitado" del "paradigma amplio": el primero se refiere a objetivos dentro de las "políticas para la ciencia", como 
mejorar la calidad, el alcance y la masa crítica de la investigación o tornar más atractivo el Espacio Europeo para recursos humanos altamente calificados provenientes de terceros países; el segundo se refiere a propósitos dentro de las "políticas a través de las ciencia", como mejorar la competitividad, enfrentar desafíos sociales globales y respaldar a los países menos desarrollados en la construcción de capacidades en ciencia y tecnología. En ambos casos, se trata de una estrategia destinada a superar las desventajas de la región europea respecto de otros competidores globales, como Estados Unidos o Japón. ${ }^{4}$

En relación con el "paradigma ampliado", un documento de la Unión Europea señalaba lo siguiente:

Europa puede jugar un rol más activo en el establecimiento de agendas globales y en la formulación de políticas y estrategias para ser más audible en las negociaciones internacionales. Tomar la delantera en la formulación de políticas puede ser la forma de reforzar sus bases de competitividad económica, influenciando tempranamente el diseño de regulaciones internacionales que afectan al sector privado (European Commission, 2008, p.29).

Esta preocupación por el establecimiento de agendas globales y por la competitividad ha impulsado dos tendencias en los Programas Marco. La primera es hacia una mayor concentración de recursos para objetivos mejor definidos y más acotados en las convocatorias a proyectos. La segunda es hacia un incremento en la participación de empresas: si entre el cuarto Programa Marco y el sexto se constató una caída de la participación de empresas en los consorcios de investigación, la contribución económica de la UE destinada a empresas industriales pasó del 17\% en el sexto Programa Marco al 25\% en el séptimo, en tanto que el porcentaje de contratos empresas con empresas industriales respecto de otras instituciones participantes pasó del 19\% al 30\% entre ambos Programas (European Commission, 2016). Un 48\% del incremento de la contribución económica se concentró en las diversas áreas temáticas que integran el subprograma "cooperación" y un 21\% se debió a la introducción de nuevos instrumentos del FP7 (European Commission, 2016).

Por lo tanto, a pesar de que los Programas Marco incluyen instrumentos para financiar proyectos destinados a enfrentar desafíos globales (como el cambio climático) o a mejorar capacidades de investigación y resolver problemas de países en desarrollo (como las enfermedades endémicas), el leit motiv de la cooperación Norte-Sur de las décadas de 19701980 ha ido perdiendo fuerza frente a una política que, bajo la apariencia de "libre acceso" a instrumentos/financiamiento europeos y "beneficio mutuo", nos interpela nuevamente sobre las asimetrías (reales o potenciales) relacionadas con la definición de las agendas y la explotación (privada) del conocimiento. ${ }^{5}$

\section{Métodos}

Nuestro trabajo empírico se orienta a hacer un trabajo cualitativo acerca de las dimensiones que explican las motivaciones para enrolar grupos latinoamericanos en proyectos europeos, y el tipo de prácticas que allí se despliegan. Por lo tanto, no tiene una validez estadística, ni sus resultados son generalizables a todas las actividades de 
cooperación científica. En cambio, elegimos observar casos paradigmáticos que nos provean información con mayor detalle sobre el objeto de estudio. Por ello, la selección de los casos que se estudian con profundidad no pretenden ser representativos, sino más bien abrir la cuestión a diversas posibilidades y configuraciones propias de los actores, de sus tradiciones, sus disciplinas, sus temas de trabajo, de las posibles aplicaciones industriales etc.

El estudio se basa en el análisis de proyectos del subprograma cooperación del FP7.

Por ello, analizamos en primer lugar las políticas y los instrumentos de cooperación científica de la Unión Europea durante las últimas dos décadas, los cambios en las orientaciones para la colaboración con diversos países y, en particular, las acciones que involucran a América Latina.

A continuación extrajimos, de la base de datos CORDIS (es decir, del total de proyectos registrados en dicha base), aquellos proyectos en los que se registró la participación de, por lo menos, un grupo de Argentina, Brasil, México, Chile o Colombia. En segunda instancia, hemos organizado esa muestra por área temática y seleccionado aquellas áreas en las que América Latina tiene mayor tradición de investigación (SALUD y KBBE) ${ }^{6}$ y otra que, a diferencia de las anteriores, implica un tipo de investigación sobre el terreno (por contraposición a la investigación de laboratorio) destinada en general al estudio de sistemas globales (ENV). Posteriormente, se seleccionaron entre cinco y seis proyectos de cada una de las tres áreas, que cumplieran con los siguientes requisitos: que estuvieran financiados con el esquema "proyectos colaborativos"7 y que abordaran tópicos diferentes dentro del área, de modo de tener representados proyectos con diversas orientaciones.

Las características de los proyectos seleccionados han sido estudiadas a través de dos mecanismos. En una primera instancia, se ha explorado el sitio web de cada proyecto (17 en total) donde constan los coordinadores, los partners de distintos países e instituciones, la descripción general del proyecto (incluyendo los objetivos de cada work package), las actividades realizadas y los resultados obtenidos. En una segunda instancia, realizamos una entrevista semi-estructurada con cada uno de los coordinadores de los proyectos seleccionado, en la que se apuntó a indagar las siguientes cuestiones:

a. Origen del proyecto (coordinador u otro grupo) y participación de los grupos latinoamericanos (GL) en el proceso de formulación del mismo.

b. Motivación para convocar específicamente a ese/esos GL: ventajas frente a la evaluación, existencia de recursos humanos altamente capacitados, acceso a recursos de la región latinoamericana (especímenes, poblaciones), conocimiento de técnica o temas específicos, aporte de fondos de contraparte etc.

c. Distribución de los work packages.

d. Tipo de actividades que desarrolló el/los GL: rutinarias, técnicas innovadoras, puesta a punto de nuevas técnicas y equipamientos, recolección de información o datos, desarrollo teórico, desarrollo de nuevos procesos o productos, transferencia al sector productivo.

e. Resultados obtenidos: publicaciones conjuntas, registro de patentes, nuevas regulaciones o normativas.

f. Rol y características de las empresas que integraron el consorcio. 
g. Negociaciones relacionadas con la propiedad intelectual de los posibles resultados.

h. Beneficios para el GL: formación de recursos humanos, apertura de nuevas líneas de investigación, acceso a fondos, contacto con líderes científicos internacionales e integración a redes globales, solución de necesidades sociales, creación de nuevas empresas.

\section{Resultados y análisis}

Para concentrar el análisis, organizamos los resultados en dos categorías principales: motivaciones de los líderes europeos para enrolar a los grupos latinoamericanos, y actividades desarrolladas por estos últimos, dentro de la división interna del trabajo, así como los beneficios obtenidos.

\section{Motivaciones para enrolar grupos latinoamericanos}

Lo primero que observamos en las respuestas de los líderes europeos en relación con las motivaciones para incluir a grupos latinoamericanos en los consorcios es que, por regla general, existe una alta valoración de la calidad científica de los científicos de América Latina convocados. Así lo afirmaron todos los líderes europeos entrevistados, como primera justificación. Ello no debe sorprendernos porque, tal como ya lo señalan algunos textos anteriores (Kreimer, 2006), los grupos de élite latinoamericanos muestran una alta correlación entre su grado de internacionalización y su grado de prestigio relativo en el medio local. Dicho de otro modo, los líderes científicos latinoamericanos construyen su prestigio en parte en su desempeño local, pero sobre todo haciendo valer localmente sus relaciones internacionales. Esto proviene de dos fuentes: por un lado, porque una parte de los líderes latinoamericanos han pasado parte de su formación en laboratorios de élite localizados en los países más avanzados (Kreimer, 2012). Así, suelen conservar lazos importantes con dichos grupos, lo que les ofrece un acceso más fácil a las redes de investigación internacionales. De hecho, la mayor parte de los líderes europeos entrevistados declararon que ya conocían a los líderes latinoamericanos antes de enrolarlos en las redes europeas. Por otro lado, las instituciones de promoción y evaluación de las carreras científicas a nivel latinoamericano (generalmente conducidas por investigadores prestigiosos) otorgan un mayor peso relativo a las vinculaciones, cooperación y obtención de fondos de agencias internacionales, lo que va generando una especie de retroalimentación puesto que a mayor internacionalización le va correspondiendo un mayor prestigio relativo.

Incluso, en el pasado, cuando aún la publicación de artículos científicos en revistas internacionales con referato no se había institucionalizado como una medición estándar de la calidad científica, parte del prestigio local se ganaba a partir del reconocimiento de pares internacionales. ${ }^{8}$ En la actualidad, participar en proyectos internacionales - y/o financiados con fondos internacionales - es una fuente de prestigio importante en todos los países, pero ello es más evidente en los de desarrollo científico intermedio.

Hay, sin embargo, diferencias sustantivas en cuanto a los estímulos para enrolar grupos latinoamericanos. Hemos identificado cuatro tipos de motivación/relación: 
a) La inclusión de latinoamericanos como condición para la obtención del subsidio

Es el caso de SALUD-5; KBBE-6 y ENV-6. En estos casos cabe preguntarse por la motivación en la elección de esos grupos en vez de otros, pero no por qué incluir a un grupo latinoamericano. La respuesta aquí parece venir de dos fuentes diferentes: la primera, de tipo técnico-cognitiva, como las capacidades adicionales que pueden proveer los grupos latinoamericanos invitados, o el acceso a recursos cognitivos, como la disponibilidad de cepas específicas, el acceso a enfermos etc. La segunda es de orden sociológico y refiere a los vínculos establecidos entre los partenaires en el pasado, los lazos de familiaridad y de confianza e, incluso, los paradigmas científicos compartidos que son frecuentemente evocados por los entrevistados.

\section{b) Consorcios que trabajan sobre cuestiones latinoamericanas}

Es el caso de la mayor parte de los proyectos de salud, que incluyen enfermedades tropicales (como la enfermedad de Chagas): SALUD-1, 4 y 5. En este caso la pregunta adicional que debemos formular es por qué Europa decide financiar la investigación sobre temas que no afectan (o afectan solo marginalmente) su propio contexto. Aquí también encontramos dos niveles de respuesta diferente: el primero refiere a lo que la investigación sobre cuestiones (enfermedades) locales puede aportar a la comprensión de fenómenos de alcance más fundamental. En este sentido, por ejemplo, la investigación sobre búsqueda de blancos para atacar a los agentes causales de esas enfermedades puede dar pistas sobre mecanismos biológicos o fisiológicos fundamentales que serían extrapolables a otros aspectos cognitivos por fuera de los casos estudiados. Además, la prueba con moléculas novedosas puede generar, en asociación con empresas del sector farmacéutico, conocimientos aplicables a enfermedades de tipo universal, autonomizándose de las cuestiones exclusivamente locales.

El segundo nivel de respuesta refiere al resultado de los procesos de globalización y de las corrientes migratorias: algunas enfermedades clásicamente "tropicales" se fueron extendiendo hacia otras regiones y generan nuevos problemas frente a los cuales hay muy poco conocimiento acumulado. Este es el caso, por ejemplo, de la enfermedad de Chagas en España, Francia o en los estados de Texas y California en Estados Unidos. En estos casos, además del prestigio que los investigadores latinoamericanos puedan detentar, se suma una expertise específica en relación con un objeto de estudio, y el acceso exclusivo a recursos indispensables para la investigación, como los pacientes, o las diversas cepas de parásitos $\mathrm{u}$ otros organismos.

\section{c) Consorcios que investigan problemas globales, con manifestaciones específicas en diversos contextos}

Aquí se necesitan diversos puntos de observación localizados en contextos diferentes. Este es el caso más frecuente en los proyectos relacionados con el medio ambiente y, en nuestro estudio, en los consorcios ENV-1, 2 y 4 . En estos casos, los fenómenos que se investigan responden a fenómenos de orden más general, como el cambio climático, y tienen manifestaciones en algunos sitios geográficos bien localizados, como la concentración de 
mercurio en los ríos o las consecuencias del derretimiento de los glaciares. En estos casos, la incorporación de grupos latinoamericanos responde a dos modalidades complementarias: la primera es la necesidad de recolectar datos en puntos de observación específicos que generen una "mirada global" del problema. En estos casos, los grupos latinoamericanos deben tener un acceso a esos recursos - ríos, glaciares, diversas especies de fauna o flora etc. - y contar con la capacidad técnica para generar datos en forma estandarizada según los protocolos establecidos por el conjunto del consorcio, ya que aquí el uso de la metodología es clave para la homogeneidad de los datos recogidos.

La segunda modalidad se refiere a que los grupos latinoamericanos deben poder movilizar recursos cognitivos - y también técnicos - como el conocimiento de sus contextos específicos y, en particular, poder utilizar equipamientos que les permitan realizar un trabajo homogéneo con el de los otros sitios de observación.

Luego, desde la coordinación del programa se procesan los resultados (en algunos proyectos ya hay un primer grado de procesamiento local) y se elaboran los productos, que pueden tener la forma de protocolos de intervención, recomendaciones de política (a organismos internacionales, a la Unión Europea o a gobiernos nacionales) y también bajo el formato de artículos científicos o eventualmente de transferencia a empresas privadas, si bien en el caso de los proyectos de medio ambiente su participación suele ser menor que en otros campos temáticos.

d) América Latina resulta un contexto importante - y a veces indispensable - para la observación/ experimentación

Es el caso del desarrollo de organismos genéticamente modificados (OGM) que corresponden al consorcio KBBE-1. Si bien en Europa están autorizados los ensayos en laboratorio, es prácticamente imposible realizar ensayos de campo a una escala importante. En cambio, en América Latina existen muy pocas restricciones para ensayos de OGMs, siempre que se respeten algunas normas técnicas básicas. En este sentido, los consorcios que desarrollan actividades que están prohibidas o son muy difíciles en Europa deben conseguir, imperiosamente, un terreno fuera de su región donde poder desarrollar dichos ensayos. Algo similar ocurre en los consorcios KBBE-4 y SALUD-1, en donde están implicadas pruebas clínicas con pacientes que, si bien no están prohibidas en la Unión Europea, tienen regulaciones mucho más rígidas que en Argentina y Brasil, donde se han desarrollado dichos ensayos en ambos consorcios, con menores control y, por lo tanto, mayor celeridad en la obtención de resultados.

\section{Tipo de actividad de los grupos latinoamericanos y beneficios obtenidos}

En la muestra de los consorcios de investigación que hemos seleccionado para nuestra indagación, los tipos de actividades realizadas por los grupos latinoamericanos que prevalecen, en todos los campos temáticos, son la recolección y sistematización de datos y el trabajo técnico, ya sea rutinario o innovador. Ello nos refiere a un tipo de inserción específica en la planificación de la investigación que es consistente con las causas que implican el reclutamiento de dichos grupos: como vimos, en la mayor parte de los proyectos predominan las razones referidas a los sitios de observación de fenómenos, sean estos 
específicos de América latina (aunque con intereses globales), o las manifestaciones locales de fenómenos de un alcance mucho amplio.

A ello se agregan dos datos muy importantes: por un lado, en la mayoría de los casos los grupos latinoamericanos fueron invitados a participar de los consorcios una vez que las grandes líneas del plan de trabajo ya estaban diseñadas y, por lo tanto, su participación en el diseño metodológico y en la distribución de tareas ha sido muy escasa. Hay solo tres excepciones: los casos caso de SALUD-1 y 4 y KBBE-6, donde los grupos latinoamericanos participaron activamente del diseño.

Por otro lado, solo en dos de los consorcios analizados los grupos latinoamericanos estuvieron a cargo de la coordinación de un work package, es decir de la administración de una parte de los recursos asignados al proyecto. Se trata de SALUD-4 y KBBE-6 (que son también dos de los proyectos en donde los grupos participaron del diseño general de la investigación) y, en ambos casos, se trata de grupos de Brasil, muy prestigiosos, con una muy fuerte tradición en investigación y gran visibilidad, lo que los constituye en espacios de referencia internacional (Fiocruz y Embrapa).

En relación con la participación de las empresas, en todos los consorcios analizados, (con la excepción de 3: ENV-1, ENV-4 y ENV-5) participan firmas de países europeos y, en dos proyectos, participan empresas latinoamericanas (una chilena y una argentina, respectivamente). Debemos señalar que, según disposiciones de la Unión Europea, las empresas que participan de los consorcios deben ser pequeñas y medianas. Según las declaraciones de los directores de los consorcios, ninguna de las empresas participantes en estos consorcios ha hecho aportes de fondos específicos para la investigación, sino que sus aportes son más bien "en especie", por ejemplo, a través de la provisión de compuestos químicos, capacidad de procesamiento de datos, actividades de marketing y difusión o equipamientos específicos. Intentamos indagar en todos esos proyectos sobre los acuerdos para la propiedad industrial y para la explotación de los resultados de las investigaciones, pero resultó imposible, son disposiciones reservadas.

Dicho esto, debemos destacar algunas particularidades según los diversos campos temáticos-disciplinarios:

De los proyectos de salud, dos están orientados a la enfermedad de Chagas, una endemia propia de América Latina, y cuyos grupos más activos en el mundo corresponden a investigadores de Brasil, Argentina y, en menor medida, Colombia. En estos consorcios es donde la participación en el diseño de las investigaciones, y en las diferentes etapas es realmente significativo y uno de ellos (SALUD-4) es de los poquísimos casos en que un grupo latinoamericano administra un work package. De hecho, es también uno de los pocos que señala que el principal beneficio para el grupo es en términos económicos, ya que "los recursos que administran son muy importantes".

Asimismo, todos los proyectos de salud implican la participación de empresas, por lo general laboratorios farmacéuticos. Sin embargo, una sola de esas empresas es latinoamericana. Así, cuando intentamos indagar quién se haría cargo de la industrialización de los resultados que se obtendrían como resultado del consorcio, descubrimos que sería un laboratorio industrial europeo quien estaría a cargo de la producción de nuevos fármacos, mientras que el latinoamericano sólo se ocuparía de la distribución. 
Por otro lado, en el área de salud, casi todos los grupos latinoamericanos tienen vínculos con pacientes y solo en el caso de SALUD-2, el grupo latinoamericano (chileno, en este caso) aporta la propiedad de compuestos de interés biológico y la capacidad de desarrollar análisis de big data.

No debemos pensar que en el campo de la salud las tareas de "recolección" y de "sistematización" de datos son actividades meramente técnicas sin contenido científico: al tratarse de desarrollos experimentales, las capacidades científicas resultan fundamentales. Sin embargo, se trata de actividades subordinadas a una capacidad centralizada de procesamiento de la información, siempre localizada en alguno de los centros europeos.

En KBBE la situación es bastante similar: en general se refiere a procesos de investigación en agricultura y agroindustria, donde son determinantes la disponibilidad de recursos cognitivos sobre el medio local, y las fuertes tradiciones de investigación de los grupos latinoamericanos. Así, dos de los proyectos se orientan a hacer ensayos de campo en sus propios países, ya que en un caso ellos están prohibidos en Europa (KBBE-1) y en el otro las regulaciones son más laxas en nuestra región (KBBE-4). Otros grupos también se dedicaron a hacer ensayos clínicos (KBBE-5) o de campo (KBB-6), actividades que son claramente predominantes.

Curiosamente, en KBBE, los grupos que desarrollan actividades de mayor complejidad científica son ambos de Chile: uno es un centro veterinario dedicado a la producción de vacunas (KBBE-3), y el otro es experto en una técnica de referencia internacional para la valoración de biorresiduos (KBBE-2).

En el campo de la investigación sobre medio ambiente, que tiene una menor tradición relativa en América Latina en relación con los dos campos anteriores (salud y agricultura), todos los grupos identificados realizan actividades de recolección de información y producción de datos, y en todos los casos según protocolos ya establecidos. Los datos generados por los grupos latinoamericanos se orientan a objetivos diferentes: sistematizar la concentración de mercurio en las aguas de diversas regiones (ENV-1); utilizar modelos globales con puntos de observación específicos (ENV-2 y ENV-4); generar modelos conceptuales (ENV-3); o proponer políticas y herramientas de intervención (ENV-6). Sin embargo, la participación de los científicos de nuestra región en esos objetivos globales es muy baja o inexistente, ya que a menudo sus prácticas se detienen luego del procesamiento y la sistematización de los datos generados.

En cuanto a los beneficios de la investigación para los grupos latinoamericanos, los hallazgos son concluyentes: el mayor beneficio se observa en términos del aumento de las publicaciones internacionales. A ello se le agrega la posibilidad de interactuar con grupos de investigación prestigiosos en el plano internacional. Contrariamente a lo que suponíamos, la obtención de fondos no aparece como el primer beneficio para la mayoría de los grupos latinoamericanos, según lo que declaran los coordinadores europeos. Ello puede deberse a diversas razones: primero, que al tratarse de grupos de élite, ya tengan cubiertas sus necesidades fundamentales de financiamiento; segundo, que haya beneficios intangibles, como acceso a metodologías de punta, a compartir bases de datos internacionales, o a otros conocimientos tácitos; tercero, a que si bien la magnitud de recursos generales de los consorcios sea relativamente importante, la parte proporcional que reciben los 
latinoamericanos (con las pocas excepciones en que son, a su vez, administradores) no se perciba como significativa, en términos comparados con otros beneficios.

\section{Elementos para una tipología}

Para finalizar, y en función de todo lo anterior, queremos proponer una tipología preliminar de las diversas modalidades de inserción de los grupos latinoamericanos en consorcios internacionales, sustentada en el trabajo empírico. Para ello, definimos cuatro tipos de participación, y los mostramos tomando como ejemplo concreto a algunos de los consorcios analizados.

Tipo 1: Participación en función de los recursos locales en América Latina indispensables para la investigación.

Tipo 2: Participación por "subcontratación" para contribuciones específicas a una investigación global o para generar regulaciones.

Tipo 3: Participación en función de la reputación internacional del grupo de América Latina, o conocimientos específicos necesarios para la investigación.

Tipo 4: Participación en función de la posibilidad de hacer ensayos (clínicos, agrícolas) menos regulados en América Latina que en Europa.

Veamos brevemente cada una de estas modalidades:

Tipo 1: BERENICE (Benznidazol and Triazol Research Group for nanomedicine and Innovation on Chagas Disease).

Consorcio coordinado por un grupo catalán: pretende testear el perfil tóxico del benznidazol, única droga existente para el tratamiento de Chagas.

El grupo brasileño (Fiocruz y Ouro Preto) se dedica al diseño y puesta en marcha de ensayos clínicos. Según el coordinador, "son una pieza fundamental: el ensayo está basado exclusivamente en los datos producidos por los grupos brasileños (con enfermos de Chagas)". Se ocupan también de los modelos in vivo. El grupo argentino (ANLIS) se ocupa de testear las nuevas moléculas alternativas al benznidazol y su contribución es también "indispensable, gracias a su experiencia con T. cruzi". ${ }^{9}$

A pesar de su importancia aparente, los grupos de AL no participaron en absoluto en la definición del proyecto (objetivos, métodos o distribución de tareas).

Tipo 2: VIROCLIME (Impacts of Climate Change on the Transport, Fate and Risk Management of Viral Pathogens in Water).

Consorcio coordinado por un grupo inglés: analiza la polución de las aguas de los ríos por diferentes tipos de virus, como consecuencia del cambio climático.

El proyecto estudia diversos ríos europeos y quiere contrastar con una región tropical (estudios en la cuenca del Amazonas. El grupo brasileño (UFRJ) fue convocado porque es "muy respetado internacionalmente" pero sobre todo porque "era necesario (y oportuno) tener una localización tropical y ellos tienen acceso al Amazonas". Su trabajo fue "tomar muestras según los protocolos, hacer las mediciones - que es un trabajo delicado - y generar los informes correspondientes". 
El grupo coordinador ya había tenido dos pedidos de la Comisión Europea (y dos proyectos europeos) para elaborar un policy report sobre la epidemiología de los ríos y las mediciones de concentración viral.

Tipo 3: EPIMIRNA (Micro RNA in the pathogenesis treatment and prevention of Epilepsy)

Consorcio coordinado por un grupo irlandés: encontrar un tratamiento gracias al micro RNA (pequeñas moléculas celulares) para casos en los cuales los medicamentos tradicionales no son eficaces para tratar la epilepsia.

El grupo brasileño (Campinas) fue convocado porque es "muy conocido internacionalmente, con publicaciones importantes en el campo de la epilepsia. Sobre todo, son muy fuertes en los aspectos genéticos y lo que queríamos en este proyecto era mirar la genética de un tipo particular de gen en la epilepsia. Ese grupo brasileño tiene realmente competencias increíbles en el campo de la genética de la epilepsia".

Por otro lado, para el consorcio era muy útil ensayar con "otras poblaciones genéticamente hablando - fuera de Europa ... y es por eso que imaginamos hacer ensayos en USA y en Brasil" (el grupo está ligado a una clínica en São Paulo donde disponen de enfermos). Sin embargo, el trabajo genético fuera de Europa fue coordinado por un investigador americano (Columbia University).

Tipo 4: AMIGA (Assessing and Monitoring the Impacts of Genetically modified plants on Agroecosystems).

Consorcio coordinado por un grupo italiano: el objetivo es evaluar el impacto ambiental y económico posible del cultivo de OGMs. Uno de los que se evalúa en este proyecto es el de la papa transgénica, tema en el cual el grupo argentino (INTA) trabajó durante varias décadas. La tarea del grupo argentino es en particular trabajar para la validación de la metodología de control en las áreas en las que los OGMs son cultivados a gran escala. Una primera validación de los métodos se prevé en el Reino Unido, pero es necesario probarlo a una producción de gran escala lo que está permitido en AL pero no en Europa.

\section{Consideraciones finales}

A través del análisis empírico pudimos constatar que, mientras que las relaciones científicas se van complejizando, y la participación de grupos de investigación latinoamericanos en consorcios internacionales es cada vez mayor, la estructura básica de esas relaciones sigue organizándose en torno de modalidades de la "integración subordinada": las actividades que más emprenden los investigadores de América Latina en la división del trabajo en el interior de los consorcios de investigación es la producción, organización y sistematización de datos. La expansión de las tecnologías para compartir tanto materiales como resultados de la investigación no parece haber modificado la estructura de relaciones anteriores. Mientras tanto - con pocas excepciones - los grupos europeos tienden a concentrar el diseño de las investigaciones, tanto en términos teóricos como, sobre todo, metodológicos, así como la capacidad de centralizar los datos generados y la de producir interpretaciones conceptuales. 
En la medida en que casi todos los grupos de investigación latinoamericanos (con las excepciones mencionadas) son invitados a participar de los consorcios una vez que el diseño de la investigación ya ha sido establecido, la capacidad para orientar los resultados de esos trabajos hacia necesidades o un uso potencial en América Latina resulta extremadamente débil.

Por otro lado, y tal como señalamos en el acápite anterior, en casi todos los consorcios participan empresas europeas, generalmente medianas. Aunque por razones de confidencialidad no pudimos acceder a los acuerdos sobre propiedad industrial y explotación de resultados, es bastante plausible deducir que dichos acuerdos implican que, de obtenerse resultados explotables comercialmente, ellos serán aprovechados en primer lugar por las empresas participantes de dichos consorcios y, enseguida, por otras empresas europeas. Así, la industrialización de los conocimientos - si tiene lugar - habrá de desplegarse prioritariamente en el continente europeo.

Retomemos los dos paradigmas que forman parte de los discursos explícitos por parte de la Unión Europea, el "limitado" y el "ampliado". En función de nuestra investigación podemos observar que la puesta en práctica del paradigma limitado - es decir, el propósito "de mejorar la calidad, el alcance y la masa crítica de la investigación" - implicó la creciente participación de investigadores latinoamericanos, quienes aportan capacidades de investigación especializada en campos temáticos específicos, que se suman a los recursos humanos específicamente europeos. Ello ocurre, a diferencia del pasado, sin que se produzcan migraciones científicas permanentes o de larga duración, sino que los grupos latinoamericanos trabajan desde sus países y aportan para ello, además, las infraestructuras ya existentes allí.

Por otro lado, se genera un efecto de "proximidad cultural" y de comunidades ampliadas, entre pares de ambas regiones, que alimenta la cantidad de conocimientos disponibles para un uso potencial por parte de Europa.

Vale la pena considerar el "paradigma ampliado", es decir, el plano de las políticas y al propósito de mejorar la competitividad, enfrentar desafíos sociales globales y respaldar a los países menos desarrollados en la construcción de capacidades en ciencia y tecnología. Digamos rápidamente que, dadas las características del importante desarrollo relativo de los grupos científicos de los países considerados, el fortalecimiento de las capacidades de esos países sin dudas ocurre como fruto de la cooperación analizada, pero no es un elemento determinante (como lo sería en el caso de países con menor desarrollo). En cambio, es claro que la naturaleza de los proyectos considerados y sus dinámicas permiten fortalecer una visión global por parte de la Unión Europea, tanto a través de "puntos de observación" de diversos fenómenos globales en América Latina (como el cambio climático), como la posibilidad de hacer ensayos en terrenos con menos regulaciones, o aún por la posibilidad de generar conocimientos de cuestiones locales que completen una visión más amplia o que contribuyan a generar regulaciones en Europa.

Llegado a este punto, podríamos plantear la hipótesis de una "interdependencia funcional" entre las políticas europeas de estímulo para la participación de científicos extra-europeos y las élites latinoamericanas: mientras que la UE logra ampliar la cantidad de investigadores que se dedican a abordar cuestiones específicas de interés europeo, las élites latinoamericanas 
encuentran un modo de formalizar sus vínculos internacionales, incrementar sus publicaciones y, en términos generales, la visibilidad internacional de sus trabajos.

Adicionalmente, vale la pena mencionar el papel de las instituciones de política, promoción y administración de las carreras científicas latinoamericanas. Por regla general, estas instituciones (universidades o consejos nacionales, como CONICET de Argentina, CONACYT de México, CNPq de Brasil o COLCIENCIAS en Colombia) suelen estimular la cooperación internacional bajo la creencia (aún con matices que no podemos analizar aquí) de que "cuanto mayor sea el grado de cooperación internacional de sus investigadores, mayor será el beneficio para el desarrollo de la ciencia local". Así, el cosmopolitismo como un valor intrínseco de las comunidades científicas locales parece haber atravesado las últimas décadas sin que se promueva (con la excepción de un breve período durante el gobierno de Lula en Brasil, y algunas iniciativas contradictorias de la última década en Ecuador) una reflexión crítica, desde el punto de vista de las políticas de ciencia y tecnología, acerca del papel relativamente subordinado que ocupan los investigadores latinoamericanos en las redes colaborativas promovidas desde los países más desarrollados. Ello se expresa mayormente, como mostramos, en la definición de las agendas, en la selección de técnicas de investigación, en cierta distribución asimétrica de las tareas y en el uso potencial de los conocimientos que se producen, dada la histórica debilidad de las sociedades latinoamericanas en la industrialización local de los conocimientos. Por lo tanto, la mera participación de grupos de la región en redes internacionales es valorado en la evaluación de las carreras científicas como un elemento favorable per se, que rara vez indaga acerca de las características y beneficios que dicha participación implica.

Finalmente, esperamos que la tipología - preliminar - propuesta constituya un punto de partida para profundizar el análisis sobre la participación de grupos latinoamericanos en consorcios internacionales y, más genéricamente, acerca de algunas modalidades sobre las que se estructuran las relaciones entre los centros hegemónicos de producción de conocimientos y los contextos semi-periféricos. También es nuestra aspiración que la tipología pueda ser enriquecida con nuevas investigaciones empíricas, que tomen en cuenta, además de la cooperación con Europa, los vínculos financiados por las diversas agencias de los Estados Unidos, y también por modalidades de cooperación menos formalizadas, para observar si siguen un patrón similar de aquellas que observamos aquí o si es posible identificar modalidades distintas.

\section{NOTAS}

${ }^{1}$ La definición de cooperación "Norte-Sur" es engañosa, puesto que traslada en forma mecánica una definición geográfica a las capacidades de cada contexto regional. En términos estrictos, México está en el Norte, y Australia en el Sur. Mencionamos la expresión porque sigue estando fuertemente anclada en los discursos, pero la definición "periférico" o "no hegemónico" nos parece mucho más adecuada a nuestro objeto.

${ }^{2}$ En este artículo mostramos los resultados de un cuestionario enviado a 900 investigadores latinoamericanos - participantes en proyectos europeos correspondientes a los Programas Marco sexto y séptimo - en donde se los interrogó sobre 21 cuestiones diferentes.

${ }^{3}$ Esta cifra solo incluye la participación de grupos latinoamericanos en proyectos de investigación y no en los proyectos de movilidad de investigadores. 
${ }^{4}$ En efecto, en lo referido a los recursos humanos, la cantidad de investigadores en relación con la población económicamente activa era menor en Europa que en Japón y en Estados Unidos: en 2003 esa relación era de 5,4 por 1.000 en UE frente a casi el doble en EEUU y en Japón (9 y 10,1 por 1.000 respectivamente). Por supuesto, hay importantes diferencias en el interior del espacio europeo (Muldur et al., 2006).

${ }^{5}$ Para dar una idea de una de las asimetrías estructurales, señalemos que en países como la Argentina y Brasil, la participación de instituciones privadas con fines de lucro, sumada a la de pequeñas y medianas empresas, representó alrededor del 14\% del total de participaciones en el PM7.

${ }^{6}$ Tomamos la sigla en inglés, que significa "Knowedge-based bio-Economy", que puede traducirse como la "Bioeconomía basada en el conocimiento", y que grosso modo incluye proyectos de biotecnología aplicada a la agricultura, entre otras orientaciones.

${ }^{7}$ Nos concentramos en esquemas que respaldan proyectos estrictamente de investigación, descartando el financiamiento para la conformación y/o consolidación de redes internacionales, donde la participación latinoamericana es menos relevante.

${ }^{8}$ Para casos históricos de este proceso de construcción externa de capital científico, ver Romero (2004), Cukierman (2007), Buch (2006) y Kreimer (2016), entre otros.

${ }_{9}$ En esta y otras declaraciones emitidas por coordinadores de grupos, los nombres no pueden ser mencionados debido a acuerdos previos.

\section{REFERENCIAS}

ADAMS, Jonathan.

Collaborations: The fourth age of research.

Nature, v.497, p.557-560. 2013.

ADAMS, Jonathan.

Collaborations: The rise of research networks.

Nature, v.490, p.335-336. 2012.

ANDERSON, Melissa S.

International research collaborations: anticipating challenges instead of being surprised. In: The Europa world of learning. London: Routlegde. p.14-18. 2011.

BEAVER, Donald.

Reflections on scientific collaboration (and its study): past, present, and future. Scientometrics, v.52, n.3, p.365-377. 2001.

BEIGEL, Fernanda.

Current tensions and trends in the world scientific system: alternative circuits and new forms of peripheriality. Current Sociology, v.62, n.5, p.617-625. 2014.

BOEKHOLT, Patries et al.

Drivers of international collaboration in research. Amsterdam: Technopolis Group. 2009.

BOZEMAN, Barry; CORLEY, Elizabeth. Scientists' collaboration strategies: implications for scientific and technical human capital. Research Policy, v.33, n.4, p.599-616. 2004.

BUCH, Alfonso.

Forma y función de un sujeto moderno. Bernardo Houssay y la fisiología argentina. Buenos Aires: Editorial de la UNQ. 2006.
CETTO, Ana María; VESSURI, Hebe.

The international scientific cooperation of Latin America and the Caribbean. Unesco World Science Report. Paris: Unesco. 2005.

CORDIS.

Community research and development information service for science, research and development. Disponible en: <http://cordis. europa.eu/fp7/understand_en.html $>$. Acesso: 2009.

CUKIERMAN, Henrique.

Yes, nós temos Pasteur: Manguinhos, Oswaldo Cruz e a história da ciência no Brasil. Rio de Janeiro: Relume Dumará; Faperj. 2007.

DEVAN, Janamitra; TEWARI, Parth S. Brains Abroad. The McKinsey Quarterly, n.4, p.110. 2001.

EDLER, Jakob; FLANAGAN, Kieron. Indicator needs for the internationalization of science policies. Research Evaluation, v.20, n.1, p.7-17. 2011.

\section{EULARINET.}

Latin American participation to the 6th Framework Programme of the European Commission. Bruselas: European Commission. 2009.

EUROPEAN COMMISSION.

Directorate-General for Research and Innovation. An analysis of the role and impact of industry participation in the Framework Programmes. Luxembourg: Office for Official Publications of the European Communities. 2016. 
EUROPEAN COMMISSION.

Directorate-General for Research. Opening to the world: international cooperation in science and technology: report of the ERA expert group. Luxembourg: Office for Official Publications of the European Communities. 2008.

\section{EUROPEAN COMMISSION.}

FP7 in brief: how to get involved in the EU 7th Framework Programme for Research. Luxembourg: Office for Official Publications of the European Communities. 2007.

\section{EUROPEAN COMMISSION.}

Directorate-General for Research. Impact assessment report on the specific programme international RTD Cooperation Fifth Framework Programme (1998-2002). Luxembourg: Office for Official Publications of the European Communities. 2005.

GAILLARD, Jacques.

La coopération scientifique et technique avec les pays du sud. Peut-on partager la science? Paris: Karthala. 1999.

GAILLARD, Jacques.

North-South research partnership: is collaboration possible between unequal partners? Knowledge, Technology \& Policy, v.7, n.2, p.31-63. 1994.

GAILLARD, Jacques; ARVANITIS, Rigas. Science and technology collaboration between Europe and Latin America: towards a more equal partnership? In: Gaillard, Jacques; Arvanitis, Rigas (Ed.). Research collaborations between Europe and Latin America. Mapping and understanding partnership. Paris: Archives Contemporaines. p.1-20. 2013.

GAILLARD, Jacques; GAILLARD. Anne-Marie; ARVANITIS, Rigas.

Mapping and understanding EURO-LAC international cooperation in science and technology. EULAKS Document. Seventh Framework Programme. 2010.

GALISON, Peter; HEVLY, Bruce.

Big science: the growth of large-scale research. Stanford: Stanford University Press. 1992.

GLÄSER, Jochen; LAUDEL, Grit. Integrating scientometric indicators into sociological studies: methodical and methodological problems. Scientometrics, v.52, n.3, p.411-434. 2001.

GUSMÃO, Regina.

La implicación de los países latinoamericanos en los programas europeos de cooperación CyT con terceros países. REDES, v.7, n.16, p.131-163. 2000.
HALLONSTEN, Olof

Use and productivity of contemporary, multidisciplinary big science. Research Evaluation, v.25, n.4, 2016, p.486-495. Disponible en: <https://doi.org/10.1093/reseval/ rvw019>. Acesso en: 13 ago. 2019. 2016.

JOERGES, Bernhard; SHINN, Terry (Ed.). Instrumentation between science, state and industry. Dordrecht: Kluwer Academic Publishers. 2001.

KATZ, Sylvan; MARTIN, Ben.

What is research collaboration? Research Policy, n.26, p.1-18. 1997.

KREIMER, Pablo.

Contra viento y marea en la ciencia de la modernidad periférica: niveles de análisis, conceptos y métodos. In: Kreimer, Pablo (Ed.). Contra viento y marea: emergencia y desarrollo de campos científicos en la periferia. Buenos Aires: Editorial de CLACSO, p.9-60. 2016.

KREIMER, Pablo.

Délocalisation des savoirs en Amérique Latine:

le rôle des réseaux scientifiques. Pouvoirs Locaux, n.94, n.3, p.25-28. 2012.

KREIMER, Pablo.

¿Dependientes o integrados? La ciencia

latinoamericana y la división internacional del trabajo. Nomadas, n.24, p.199-212. 2006.

KREIMER, Pablo.

Understanding scientific research on the periphery: towards a new sociological approach? EASST Review, v.17, n.4, p.13-21. 1998.

KREIMER, Pablo; LEVIN, Luciano.

Scientific cooperation between the European Union and Latin American countries: Framework Programmes 6 and 7. In: Gaillard, Jacques; Arvanitis, Rigas (Ed.). Research collaborations between Europe and Latin America: mapping and understanding partnership. p.79104. Paris: Archives Contemporaines. 2014.

KREIMER, Pablo; MEYER, Jean-Baptiste. Equality in the networks? Some are more equal than others. International scientific cooperation: an approach from Latin America. In: Vessuri, Hebe; Teichler, Ulrich (Ed.). Universities as centers of research and knowledge creation: an endangered species? Rotterdam: Sense. p.121-135. 2008.

LEYDESDORFF, Loet; WAGNER, Caroline. International collaboration in science and the formation of a core group. Journal of Informetrics, v.2, n.4, p.317-25. 2009. 
LOSEGO, Philippe; ARVANITIS, Rigas.

La science dans les pays non hégémoniques.

Revue d'Anthropologie des Connaissances, v.2, n.3, p.334-342. 2008.

MULDUR, Ugur et al.

A new deal for an effective European research policy: the design and impacts of the 7th Framework Programme. [S.1.]: Springer. 2006.

\section{NATIONAL SCIENCE BOARD.}

International science and engineering partnerships: a priority for U.S. foreign policy and our nation's innovation enterprise. Washington: The National Academies. 2008.

NEWMAN, Mark.

The structure of scientific collaboration networks. Proceedings of the National Academy of Science of the USA, n.98, p.404-409. 2001.

OECD.

Organisation for Economic Co-operation and Development. International mobility of the highly skilled. Disponible en: <http://dx.doi. org/10.1787/9789264196087-en>. Acesso em: 13 ago. 2019. 2002.

PERSSON, Olle; GLÄNZEL, Wolfgang; DANELL, Rickard.

Inflationary bibliometrics values: the role of scientific collaboration and the need for relative indicators in evaluative studies. Scientometrics, v.60, n.3, p.421-432. 2004.

PESTRE, Dominique.

Regimes of knowledge production in society: towards a more political and social reading.

Minerva, v.41, n.3, p.245-261. 2003.

PRICE, Derek de Solla.

Little science, big science. New York: Columbia University Press. 1973.

RODRIGUEZ MEDINA, Leandro.

Construyendo periferia: un microanálisis de objetos subordinantes como tecnologías epistémicas. Sociológica, v.29, n.83, p.9-46. 2014.

ROMERO, Flor.

Manuel Elkin Patarroyo: un scientifique mondial. Inventeur du vaccine de synthèse de la malaria. Paris: L'Harmattan. 2004.
SEBASTIÁN, Jesús.

Conocimiento, cooperación y desarrollo. Revista CTS, v.3, n.8, p.195-208. 2007.

SHRUM, Wesley.

Reagency of the internet, or, how I became a guest for science. Social Studies of Science, v.35, n.5, p.723-754. 2005.

VELHO, Léa.

North-South collaboration and systems of innovation. The International Journal of Technology Management and Sustainable Development, v.1, n.3, p.171-181. 2002.

VESSURI, Hebe.

Scientific cooperation among unequal partners: the strait jacket of the human resource base. In: Gaillard, Jacques (Ed.). Coopérations scientifiques internationales. Paris: Éditions de l'ORSTOM. 1996.

WAGNER, Caroline.

The new invisible college: science for development. Washington: Brookings Institution Press. 2008.

WAGNER, Caroline.

International collaboration in science and technology: promises and pitfalls. In: Box, Louk; Engelhard, Rutger (Ed.). Science and technology policy for development, dialogues at the interface. London: Anthem Press. p.165-176. 2006.

WAGNER, Caroline; KIT WONG, Shing. Unseen science? Representation of BRICs in global science. Scientometrics, n.90, v.3, p.10011013. 2012.

WHITLEY, Richard.

Reconfiguring the public sciences: the impact of governance changes on authority and innovation in public science systems. In: Whitley, Richard; Glaeser, Jochen; Engwall, Lars (Ed.). Reconfiguring knowledge production: changing authority relationships in the sciences and their consequences for intellectual innovation. Oxford: Oxford University Press. p.3-50. 2010. 